\title{
COMMENT ON “GEODYNAMICS AS WAVE DYNAMICS OF THE MEDIUM COMPOSED OF ROTATING BLOCKS" BY A.V. VIKULIN [GEODYN. TECTONOPHYS., 2015, 6 (3), 345-364]
}

\author{
Yu. L. Rebetsky \\ O.Yu. Schmidt Institute of Physics of the Earth of RAS, Moscow, Russia
}

Recommended by G.G. Kocharyan

For citation: Rebetsky Yu.L. 2016. Comment on "Geodynamics as wave dynamics of the medium composed of rotating blocks" by A.V. Vikulin [Geodyn. Tectonophys., 2015, 6 (3), 345-364]. Geodynamics \& Tectonophysics 7 (2), 337-341. doi:10.5800/GT-2016-7-2-0211.

Для цитирования: Ребецкий Ю.Л. Комментарий к публикации А.В. Викулина «Геодинамика как волновая динамика блоковой вращающейся среды» [Геодинамика и тектонофизика, 2015, т. 6, № 3, с. 345-364] // Геодинамика и тектонофизика. 2016. T. 7. № 2. C. 337-341. doi:10.5800/GT-20167-2-0211.

\section{КОММЕНТАРИЙ К ПУБЛИКАЦИИ А.В. ВИКУЛИНА «ГЕОДИНАМИКА КАК ВОЛНОВАЯ ДИНАМИКА БЛОКОВОЙ ВРАЩАЮЩЕЙСЯ СРЕДЫ» [ГЕОДИНАМИКА И ТЕКТОНОФИЗИКА, 2015, Т. 6, № 3, С. 345-364]}

\section{Ю. Л. Ребецкий}

Институт физики Земли им. О.Ю. Шмидта РАН, Москва, Россия

В работе А.В. Викулина [Vikulin, 2015], опубликованной в журнале «Геодинамика и тектонофизика», вслед за другими работами этого автора, утверждается, что блок земной коры, двигающийся под влиянием тектонических сил (рис. $1, a$ ), должен формировать в своей окрестности дополнительное ротационное напряженное состояние.
При этом само дополнительно возникающее вращение блока является следствием меридиональной компоненты смещения и суточного вращения Земли $\left(\Omega \approx 7.27 \cdot 10^{-5}\right.$ рад/с - угловая скорость суточного вращения Земли). А.В. Викулин не дает объяснения генезиса деформирующих сил. Имеет место только указание на их взаимосвязь с суточным 
$a$

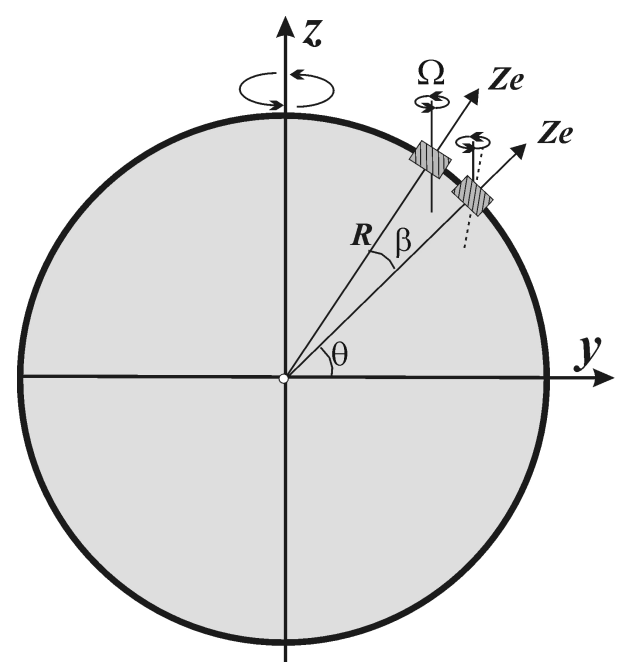

б

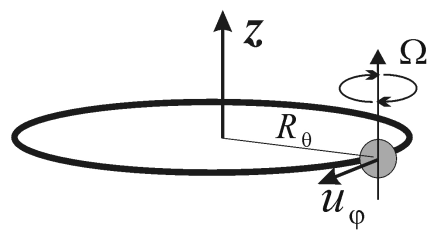

Рис. 1. Схемы, иллюстрирующие основные теоретические положения работы [Vikulin, 2015], лежащие в основе ротационных эффектов в геодинамике: $a$ - изменение положения оси собственного момента блока относительно оси на зенит в процессе его смещения вдоль меридиана по часовой стрелке (пунктир - предыдущее положение оси относительно блока); б - представление движения блока (точки) по окружности радиуса $r$ через поступательное движение $u_{\varphi}=r \Omega$ (в данном случае вдоль параллелей) и вращение $\Omega$ вокруг оси, проходящей через центр масс блока.

Fig. 1. Schemes illustrating the main theoretical concepts [Vikulin, 2015] substantiating rotational effects in geodynamics: $a$ - change in the position of the block's own moment axis relative to the zenith axis during the block's displacement along the meridian clockwise (dotted line - the previous position of the axis relative to the block); $\sigma$ - representation of the block's (point's) motion at the circle of radius $r$ by progressive movement $u_{\varphi}=r \Omega$ (in this case, along the parallels) and rotation $\Omega$ around the axis across the block's mass center.

вращением Земли. Отсутствие указания генезиса сил выглядит странно, так как в физике вращательного движения существует только три типа инерциальных сил.

В обсуждаемой работе базисом расчета ротационных напряжений является момент сил, который определяется как разность моментов двух последовательных положений блока, отстоящих вдоль меридиана на угол $\beta$. В работе [Vikulin, 2015] ничего не говорится о направлении вектора рассчитываемого момента сил, но если устремлять угол $\beta$ к нулю (смещение, отвечающее малому временному интервалу), то такой момент должен вызвать вращение вокруг оси у системы координат, представленной на рис. $1, a$.

В обсуждаемой работе приведена формула для расчета касательных напряжений в окружающем блок массиве:

$$
\sigma_{r \varphi}=4 \Omega \frac{R^{4}}{r^{3}} \sqrt{\frac{\rho G}{5 \pi}} \sin \theta \sin 0.5 \beta
$$

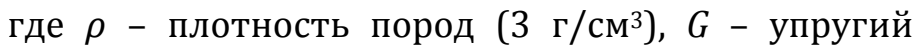
модуль сдвига (105 кГ/см3), $R$ - радиус Земли (6370 км), $r$ - радиус блока (100 км), $\theta$ - широта расположения блока. Сами напряжения $\sigma_{r \varphi}$ являются касательными, действующими в тангенциальном направлении сферической системы координат, связанной с центром блока.

Рассчитанные по формуле (1) касательные напряжения, действующие на границе блока, создают вращающий момент, который должен быть уравновешен моментом внутренних инерционных сил самого блока, но об этих силах в статье ничего не говорится. Вид выражения (1) для касательных напряжений также вызывает удивление (плотность стоит под корнем, касательные напряжения зависят от модуля сдвига?). При получении выражения (1) отсутствовала постановка задачи механики. В тексте статьи указано, что выражение (1) получено из равенства энергии упругих деформаций массива энергии, связанной с изменением момента сил (?).

В обсуждаемой работе утверждается, что уровень напряжений, который следует из выражения (1), столь значительный, что данные напряжения следует учитывать при геодинамических исследованиях и при изучении сейсмического режима.

Далее в краткой форме будет дано критическое рассмотрение поставленной в обсуждаемой работе 
проблемы. Хотя оба главных утверждения А.В. Викулина (существование собственного момента при движении блока вдоль меридиана; появление ротационных напряжений) и верны, конечный результат, полученный им (большая роль данного вида ротационных напряжений в геодинамике и сейсмике), оказался ошибочен.

Физические основы работы [Vikulin, 2015]. Действительно, любое криволинейное движение можно представить как сумму поступательного движения и вращения (рис. 1, б), и, таким образом, любой выделенный элемент земной коры имеет собственный момент, отвечающий угловой скорости суточного вращения Земли. До тех пор пока этот блок не меняет своего положения вдоль меридиана, наличие собственного момента блока не вызывает никаких геодинамических последствий, ротационные напряжения в окрестности блока не возникают.

В случае перемещения блока вдоль меридиана вращающейся Земли в нем возникают три типа инерционных сил: 1) центробежные (они действуют всегда и до начала движения блока), 2) кориолисовые, 3) гироскопические. Первые силы превратили земной шар в эллипсоид вращения, вторые при перемещении блока вдоль меридиана приводят к появлению дополнительной широтной компоненты движения. Заметим, что обе эти силы не создадут обсуждаемое вращение блока, двигающегося под действием тектонических сил. Вращение такого блока могут создать только гироскопические силы.

На рис. 2, $a$, изображено рабочее тело гироскопа в виде диска, вращающегося с постоянной угловой скоростью $\Omega$ (поддерживается внутренним источником энергии - момент сил $M_{z}$ ) вдоль вертикальной оси $Z$.

Если к внешней части гироскопа приложить момент сил $M_{x}$, создающих вращение всего гироскопа вокруг оси $x$ с угловой скоростью $\omega$, то вдоль горизонтальных штанг возникнет пара сил $F$ и $F^{\prime}$, ориентированных в разные стороны и создающих момент сил, который можно рассчитать по формуле:

$$
\mathrm{M}=\omega \times J \Omega,
$$

где $\Omega$ - вектор угловой скорости собственного вращения гироскопа, $\omega$ - вектор угловой скорости вращения гироскопа в целом за счет внешних сил, а $J$ - момент инерции тела гироскопа.

Согласно правилу Н.Е. Жуковского, массовые силы $\mathrm{f}_{\mathrm{h}}$, возникающие в теле вращающегося гироскопа (они показаны на верхней схеме гироскопа на рис. 2, б), стремятся совместить внутренний момент импульса гироскопа, вектор которого совпадает с осью собственного вращения $\Omega$, с направле- $a$

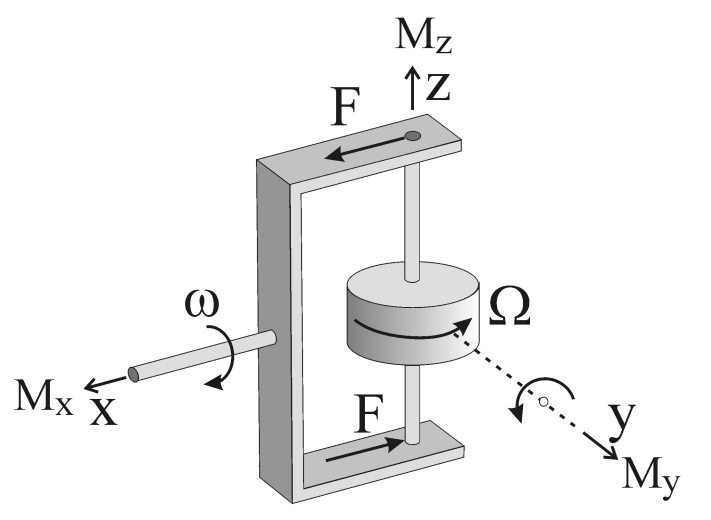

$\omega$
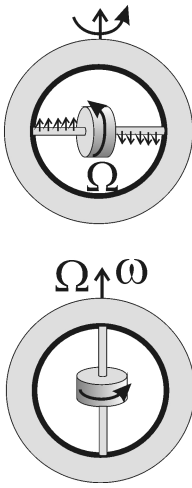

Рис. 2. Схемы гироскопа: $a$ - гироскоп (внутренний момент сил $M_{z}$ ) с приложенным к нему внешним моментом сил $M_{x}$ и возникающим моментом $M_{y}$ от пары сил $F$ и $F^{\prime}$, появление которых связано с инерционными гироскопическими силами в теле гироскопа; б иллюстрация правила Н.Е. Жуковского - тело гироскопа под действием инерционных массовых сил $\mathrm{f}_{\mathrm{h}}$ (силы показаны стрелочками одинаковой длины, но на самом деле они уменьшаются по мере приближения к центру масс гироскопа) поворачивается внутри внешней оболочки так, чтобы совместить свою ось вращения с осью вращения внешней оболочки гироскопа.

Fig. 2. Gyroscope schemes: $a$ - gyroscope (internal torque $M_{z}$ ) and external torque $M_{x}$ and emerging torque $M_{y}$ from a pair of forces $F$ and $F^{\prime}$, which occur due to inertial gyroscopic forces in the gyroscope body $\mathrm{f}_{\mathrm{h}} ; \sigma$ - illustration of N.E. Zhukovsky rule: under the impact of inertial mass forces (shown by arrows of the same length, but in fact the forces decrease closer to the gyroscope's mass center), the gyroscope's body rotates inside the gyroscope's outer shell so as to align its axis of rotation to that of the outer shell.

нием оси вынужденного поворота ( $\omega)$. Ось гироскопа стремится повернуться в плоскости хоz так, чтобы совместить оси вращения $x$ и $z$ (рис. 2, б). Эти гироскопические силы являются инерционными, действующими в каждой точке тела гироскопа. Суммируясь, они создают силы $F$ и $F^{\prime}$, показанные на рис. $2, a$. Таким образом, силы $F$ и $F^{\prime}$ не являются инерционными гироскопическими силами, но они отражают возникновение таких сил в рабочем теле гироскопа.

Следствия из проведенного анализа. Перейдем теперь к интерпретации полученного результата применительно к рассматриваемой проблеме. Если в качестве такого диска гироскопа рассматривать блок земной коры (рис. 3, a) с формой в виде куба (l - длина грани) и плотностью $\rho$, то согласно (2) для искомого момента сил можно получить следующее выражение: 
$a$

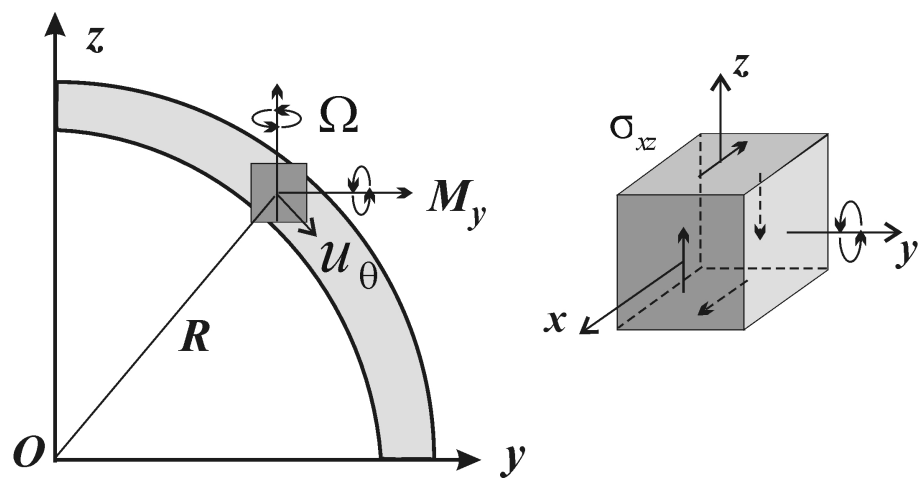

Рис. 3. Ориентация осей вращения кубического блока земной коры, двигающегося по сфере Земли вдоль меридиана $(a)$, и направления действия касательных напряжений на гранях куба (пунктирные стрелки показывают ориентацию касательных напряжений на скрытых гранях куба), создающих реактивный момент сил относительно оси у (б).

Fig. 3. Orientations of axes of rotation of a cube-shaped crustal block moving on the Earth's sphere along the meridian $(a)$, and directions of shear stresses on the faces of the cube (dashed arrows show orientations of shear stresses on the hidden faces of the cube) which generate a reactive torque relative to axis $y$ (б).

$$
M_{y}=\frac{l^{5}}{6} \rho \omega \Omega \text { при } J=\frac{l^{2}}{6} m \text { и } m=\rho l^{3} .
$$

Здесь $\omega$ - угловая скорость блока, связанная с его меридиональным движением:

$$
u_{y}=R \omega \text {. }
$$

Заметим, что вектор момента гироскопических сил направлен вдоль оси $y$, так же как это мы предположили в начале нашей заметки на основе анализа исходной информации на рис. $1, a$.

Пусть на четырех гранях такого куба, которые параллельны оси $y$, действуют касательные напряжения $\sigma_{x z}$ в направлении осей $x, z$ (рис. $\left.3, \sigma\right)$, и пусть эти напряжения создают момент сил:

$$
M_{\sigma}=2 l^{3} \sigma_{x y}
$$

Приравняв выражения для моментов сил в (3) и (5), находим:

$$
\sigma_{x y}=\frac{l^{2}}{12 R} \rho u_{y} \Omega
$$

Особо следует обратить внимание на разницу выражений (1) и (6). Согласно (6) касательные напряжения не зависят от модуля упругости среды, что вполне логично, так как они формируются изза возникающих инерционных сил (это отвечает решению статической задачи механики, когда заданы либо граничные напряжения, либо массовые силы). Напряжения линейно связаны с плотностью, скоростью тектонического движения и угловой скоростью вращения Земли.

В этой краткой заметке мы не ставили своей задачей получить значения массовых гироскопических сил $\mathrm{f}_{h}$, возникающих в блоке при его движении вдоль меридиана, так как это требует рассмотрения задачи теоретической механики в инерциальных и неинерциальных системах отсчета. Однако приближенно формулу для этих сил можно получить из теории размерности, подобрав параметры, влияющие на процесс так, чтобы выполнялось соответствие размерностей правой и левой части формулы (Г/ $\left.\mathrm{cm}^{3}\right)$ :

$$
f_{h}=k \frac{l}{R} \rho u_{y} \Omega,
$$

где $k$ - неизвестная константа.

Подставив в (6) значения параметров, отвечающих исследуемой проблеме $\left(u_{y}=10\right.$ см/год, $l=100$ км), получим, что касательные напряжения $\sigma_{x y} \approx 2.5 \cdot 10^{-13}$ бар (кГ/см²). Это очень низкий уровень напряжений. Соответственно из выражения (7) можем оценить и уровень массовых гироскопических сил $f_{h} \approx 10^{-15} \Gamma / \mathrm{cm}^{3}$, что в $10^{15}$ раз меньше массовых сил, связанных с действием силы тяжести $\left(\rho \mathrm{g}=3 \Gamma / \mathrm{cm}^{3}\right)$.

Таким образом, величины ротационных напряжений, о которых говорилось в работе [Vikulin, 2015], столь малы, что о их учете не должно идти речи. А.В. Викулину необходимо определиться с генезисом деформирующих сил и либо согласиться с предложенным вариантом (гироскопические силы), либо указать, что за силы он имеет в виду. Следует также заметить, что замена внутренних массовых сил на момент импульса внутренних массовых сил (собственный момент - в ред. А.В. Викулина) все равно требует физически правильной его оценки.

Несмотря на отрицательный результат анализа рассматриваемой в работах А.В. Викулина концепции, считаю вполне разумным поиск новых источников энергии тектонических процессов. Особо отмечу, что полученный отрицательный ответ на уровень деформирующих сил относится только к ротационным напряжениям, возникновение которых обусловлено появлением гироскопических сил. Влияние инерционных массовых сил другого генезиса в данной заметке не обсуждается. 


\section{ЛИTЕРАTУРA / REFERENCES}

Vikulin A.V., 2015. Geodynamics as wave dynamics of the medium composed of rotating blocks. Geodynamics \& Tectonophysics 6 (3), 345-364. http://dx.doi.org/10.5800/GT-2015-6-3-0185.

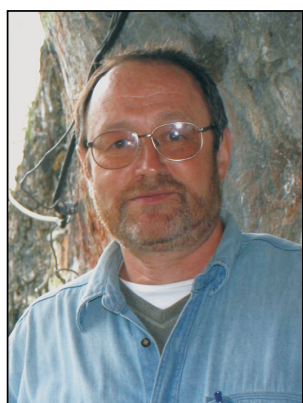

Ребецкий Юрий Леонидович, докт. физ.-мат. наук, зав. лабораторией тектонофизики им. М.В. Гзовского

Институт физики Земли им. О.Ю. Шмидта РАН

123242, ГСП-5, Москва Д-242, ул. Большая Грузинская, 10, Россия

Тел.: 84992549350; 《 e-mail: reb@ifz.ru

Rebetsky, Yuri L., Doctor of Physics and Mathematics, Head of M.V. Gzovsky Laboratory of Tectonophysics O.Yu. Schmidt Institute of Physics of the Earth of RAS

10 Bol'shaya Gruzinskaya street, Moscow D-242 123242, GSP-5, Russia

Tel.: 84992549350; \ e-mail: reb@ifz.ru 